\title{
Writing A Chemistry Education Research Article: Stepping Stone Or Stumbling Block?
}

\section{Dr Suzanne Fergus}

I was invited to write this opinion piece following a presentation at the Royal Society of Chemistry (RSC) conference on Methods in Education Chemistry Research related to translating creative teaching ideas and innovations into chemistry education research outputs. With a keen interest in learning and teaching and a solid training in organic chemistry research, my choice to combine them both and engage in chemistry education research (CER), seeking the empirical evidence to demonstrate the effectiveness (or not) of classroom or laboratory activities, would seem obvious. My professional journey has been both a stepping-stone, in terms of the positive impact of CER in deepening my pedagogic theory and stretching my ideas, yet it also has been a stumbling block. The perspective from colleagues strongly implied CER to be easier, not to mention "fluffy and vague" compared with traditional chemistry research. My experiences have been quite the contrary. I found CER if anything to be more difficult and challenging. To execute chemistry research, in for example my discipline of organic synthetic, would typically require me to consult the literature, obtain published procedures to synthesise a target compound or intermediate products and then to identify the chemicals required. I can assume that the chemicals used will react in a similar way to the published procedure. This however, cannot be assumed in education; replication is not guaranteed. An effective classroom activity with my undergraduate students or a successful doctoral research supervision strategy may not work effectively with all students in all contexts. I will outline the common pitfalls to avoid in CER, the first step in planning high quality CER and hopefully offer some reassurances that, although the transition to CER may feel like starting again within a new research discipline, it does not need to be daunting. Although my experience is in CER most of what I write here will be applicable across the disciplines in higher education.

\section{Common Pitfalls to avoid}

I found it worthwhile to appreciate initially the three key points outlined below ahead of any planned research. First, the research needs to be connected with the appropriate literature; it is essential to contextualise any teaching development within the research literature and illustrate how this informs responses to an educational issue, need or challenge. This helps establishes the background and justification for the research, which creates a good structure. Second, descriptive accounts of teaching developments and evaluations are not sufficient. Sharing a good idea does not constitute research. A lot of effort is involved in designing new teaching activities and it certainly benefits the wider chemistry education community to disseminate such ideas, but not as a research paper. Selecting the appropriate publication source from the outset will help avoid unnecessary rejection. I can recommend "What is wrong with practice papers?" (Taber, 2016) as highly useful. The third common pitfall is a lack of novelty in the research work. Simply repeating a good idea and demonstrating that it works is insufficient, as this does not go beyond what is already published and lacks originality. There are occasions when repeating research showing that the outcomes do not transfer directly to a different context merits publication as it adds new knowledge to the research topic. So 
knowing what not to do, now where to start? The question is the answer.

\section{The importance of a research question}

The first step needed to help shape innovative ideas and plans about teaching and student learning is to design appropriate research questions that can be answered empirically. Specifying aims and objectives is common in chemistry research, but crafting a research question is not. After all, it is possible to piece chemistry research outcomes retrospectively into a coherent argument, and complete any gaps not initially addressed by revisiting and repeating experiments or modifying conditions. This is definitely not readily done in education research, as the opportunity for data collection is time sensitive. Realising during the writing of a research article that missing details in your study, such as student demographic data that would enhance the results and analysis, is frustrating. It is often impossible to obtain this data retrospectively and the problem could be easily avoided during the planning of the research methodology.

The research question establishes the importance and relevance of the topic area, it connects to existing literature and influences the methods used for data collection. When presenting the results and shaping the discussion sections, the structure provided by addressing and answering the research question helps the article to flow.

It is important to stress that crafting research question(s) into something meaningful and measurable is an iterative process. 'Good' research is characterised by good research design, carried out with rigour, and significant theorisation. Generally one main research question should be addressed in a paper (with secondary but related questions). If your research project allows you to explore several distinct research questions, write several papers!

A useful taxonomy created by Hutchings (2000) to consider and think about possible research questions is shown in Figure 1. The four different question types are not mutually exclusive. "What works" questions are a good starting point to find evidence for the effectiveness of approaches. "What is" questions describe and analyse teaching and learning experiences in more detail. "Visions of the possible" focus inquiry on new goals, and that which is most important for future directions or aspirations new to the researcher. Questions on "New conceptual frameworks", as the name states, develop and shape thoughts about new theoretical frameworks and practice.

Although it may be tempting to overlook this aspect and get on with the exciting research and data collection, this really is the first stepping stone to a good quality CER. In Perneger \& Hudelson (2004), failure to specify a research question, and the discussion not providing an answer to the research question, are stated as common mistakes observed in manuscript submissions. A worksheet [adapted from Bishop-Clark \& Dietz-Uhler (2012)] to support the generation of a welldefined research question is provided in the appendix.

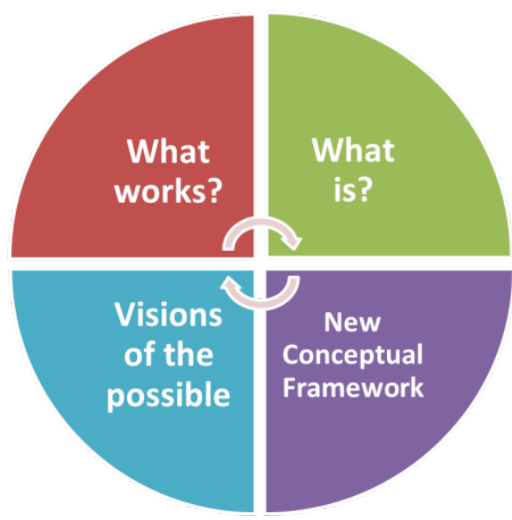

Figure 1 Taxonomy of Research Questions (Hutchings, 2000) 


\section{Developing and education research}

When it comes to planning, developing and publishing CER outputs there are a number of options available that can support the route to a journal article submission. Figure 2 illustrates some suggestions, which are not intended to be sequential. Developing a conference paper that is then written up as a blog post or article/perspective within an individual's institution is a great start to develop confidence and initiate CER writing. New Directions is an excellent external journal that provides developmental feedback and is a supportive first step to publishing a research article.

Thankfully there is a strong, supportive CER community so individuals need not feel isolated developing their efforts and establishing CER outputs. Engaging in education research and translating innovative teaching developments into CER outputs is strongly encouraged, particularly with the constructive support available. The key considerations are situating the proposed project in the research literature, justifying the research scope to ensure novelty and designing sound appropriate methodologies.

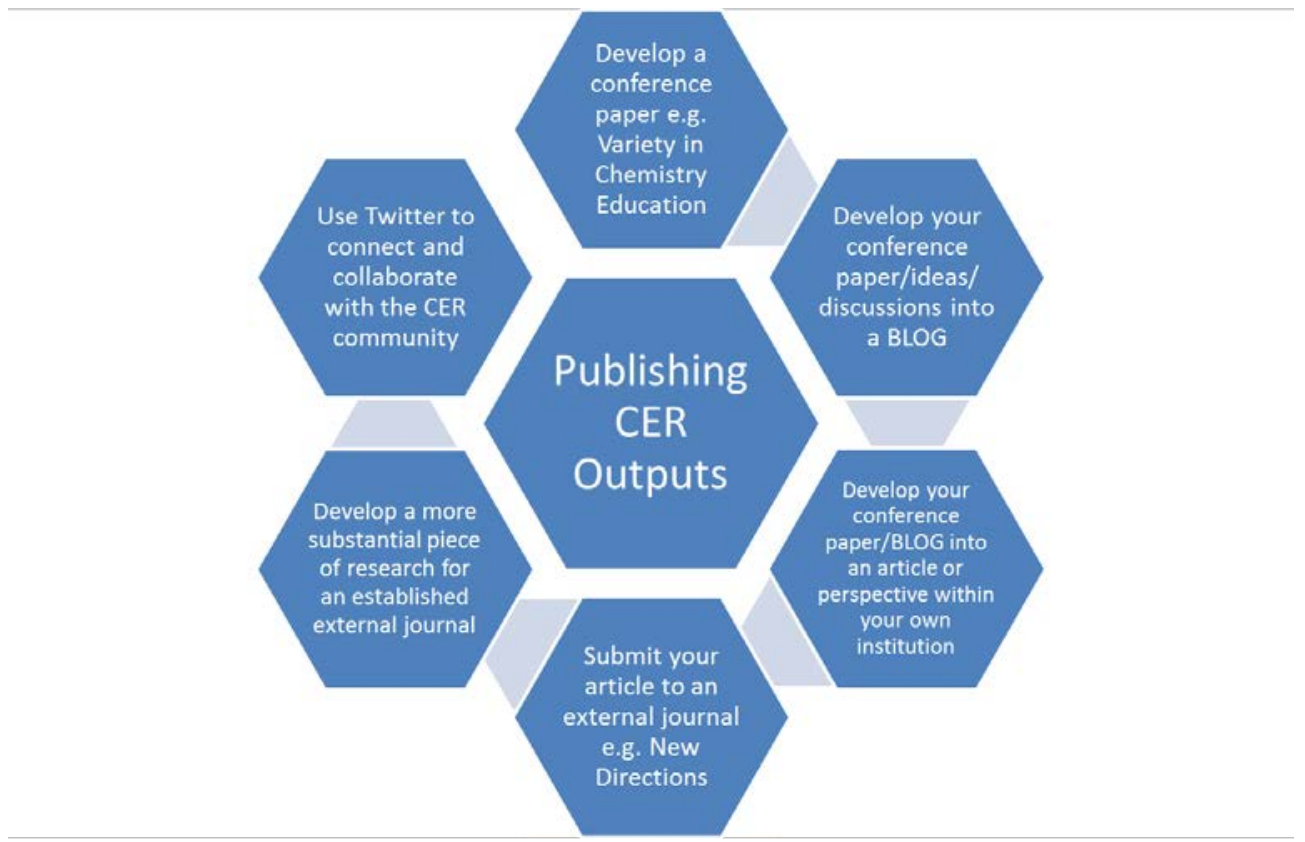

Figure 2 Planning, Developing \& Publishing your Chemistry Education Research Outputs

Dr Suzanne Fergus

2016 RSC Higher Education Teaching Award

\section{Acknowledgements}

The author would like to acknowledge Amanda Roberts and Liz White, School of Education, University of Hertfordshire for a "Planning for the development of publishable research and scholarship outcomes" framework which helped inspire Figure 2.

\section{References}

Bishop-Clark, C. \& Dietz-Uhler, B. (2012). Engaging in the Scholarship of Teaching and Learning. Stirling, Virginia: Stylus.
Hutchings, P. (2000). Opening Lines: Approaches to the Scholarship of Teaching and Learning. Menlo Park, CA: Carnegie.

Perneger T.V. \& Hudelson P.M. (2004). Writing a research article: advice to beginners. Int. J. Qual. Health Care. 16(3), 191-192. DOI 10.1093/intqhc/mzh053

Taber, K. (2016). What is wrong with 'practice' papers. Chem. Educ. Res. Pract., 17, 639-645. DOI 10.1039/C6RP90009 


\section{Appendix: Generating your Research Idea Worksheet}

The research question defines every aspect of your study - how many people you will study, the literature you will review, the research techniques and methods, the techniques to use for data collection. The following guide [adapted from Bishop-Clark, C. and Dietz-Uhler, B. (2012)] moves from a general research theme to a well-defined, clear research question. The boxes are intentionally small!

1. Identify your research theme, the general area you would like to investigate. Be sure to write in the form of a question.

2. Identify your research question. Based on this theme, can you identify a more specific research question? Be sure to write in the form of a question.

3. Identify a "too-specific" research question. Next, write your question, getting even more specific and reducing your question to an even smaller unit. Write each of your question(s) on a smaller scale, in terms as precise as possible.

4. Identify the question that makes the most sense to you and have a colleague review the question.

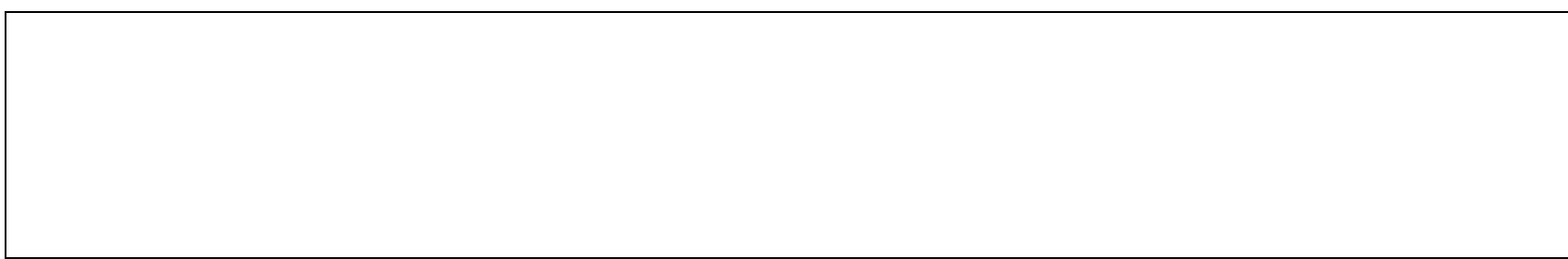


5. Identify key words that you will need to review the literature related to your research question. Consider both broad and narrow key words.

\begin{tabular}{|l|l|}
\hline Broad Key Words & Specific Key Words \\
\hline & \\
& \\
\hline
\end{tabular}

6. Following a literature review (even a quick review of key publications), are the methods used in prior work the same as or different from yours? Will your work build on work that already exists?

\begin{tabular}{|l|l|}
\hline $\begin{array}{l}\text { How will your research be the same as previous } \\
\text { work? }\end{array}$ & $\begin{array}{l}\text { How will your research be different from } \\
\text { previous work? }\end{array}$ \\
\hline & \\
& \\
& \\
\hline
\end{tabular}

7. Revise your research question, which may have changed as a result of the literature review. 